\title{
Emulsion Stability: Determination from Turbidity
}

\author{
S. R. REDDY ${ }^{1}$ AND H. S. FOGLER \\ Department of Chemical Engineering, The University of Michigan, Ann Arbor, Michigan 48109
}

Received December 19, 1979; accepted May 16, 1980

\begin{abstract}
A novel technique for studying the stability of emulsions has been developed. Owing to the fact that emulsion turbidity is a function of particle concentration and size, the stability of emulsions can be determined by measuring the change in turbidity with time. The relationship between turbidity and particle size and concentration is derived for a polydispersed emulsion. The stability of acoustically prepared emulsions determined by measuring the initial mean particle size and changes in emulsion turbidity is compared with the stability determined from scanning electron microscope measurements of the particle growth rate. The agreement between the two methods is excellent.
\end{abstract}

\section{INTRODUCTION}

A novel method for studying emulsion stability to flocculation has been developed. Emulsion stability is determined by measuring the turbidity as a function of time. This method can be applied to many oil-inwater emulsions by measuring the changes in emulsion turbidity, which is very easy and inexpensive. The turbidity of an emulsion is a function of particle size and concentration. Therefore, the change in turbidity indicates the change in particle size and concentration. In an unstable emulsion, particle concentration and size change with time due to coagulation and coalescence of particles.

The turbidity method has not been previously used for determining the stability factor, $W$, of an electrostatically stabilized emulsion. The turbidity technique has been used $(1,2)$ to determine approximately the stability of suspensions by assuming that turbidity is directly proportional to particle concentration. This assumption can lead to erroneous stability factors or aggregation rate constants as will be shown later. Dunn

${ }^{1}$ Presently at General Motors Research Laboratories, Fuels and Lubricants Department, Warren, Mich. 48090 . and Vold (1) and Bagchi and Vold (3) used this approximate method for comparing the stabilities of nonaqueous suspensions. The turbidity of suspensions containing very coarse particles varies inversely with particle size. Hiemenz and Vold (4) and Bagchi and Vold (5) used the turbidity method for determining the size of very coarse particles. The turbidity of an emulsion containing very fine and polydispersed particles is a complex function of particle size and concentration. In this study, a turbidity method is developed for determining the stability of emulsions containing polydispersed small particles. Results obtained from the turbidity method are compared with direct particle size measurements made by using a scanning electron microscope.

\section{THEORY}

The optical density or turbidity, $\tau$, of an emulsion containing monodispersed particles is given by the relationship $(6,7)$

$$
\tau=\frac{\ln \left(I_{0} / I\right)}{l}=K \pi a^{2} N,
$$

where $I_{0}$ and $I=$ the intensity of the in- 
cident and transmitted light, respectively; $a=$ the particle radius; $l=$ scattering path length; $N=$ the concentration of particles; and $K=$ the total scattering coefficient defined as

$$
K=K_{0}(a / \lambda)^{m},
$$

where $K_{0}=$ the size-independent component of the scattering coefficient, and $m=$ the exponent of the wavelength, $\lambda$, dependent on particle size and refractive index.

By measuring the turbidity at different wavelengths, the value of $m$ can be determined from the slope of a log-log plot of $\tau$ vs $\lambda$ according to the equation

$$
\tau=\text { constant }(\lambda)^{m} .
$$

For a polydispersed system, the turbidity is given by

$$
\tau=\pi \sum_{i} K_{i} a_{i}^{2} N_{i},
$$

where $K_{i}=K_{0}\left(a_{i} / \lambda\right)^{m_{i}}$.

The equation can be further simplified to a useful form by evaluating $K_{i}$ at an average particle radius, $\bar{a}$ :

Then

$$
K_{i}=K_{0}(\vec{a} / \lambda)^{m} \text {. }
$$

$$
\tau=\pi K_{0}(\bar{a} / \lambda)^{m} \sum_{i} a_{i}^{2} N_{i} .
$$

The volume fraction, $\phi$, of the dispersed phase is given by

$$
\phi=\frac{4}{3} \pi \sum_{i} a_{i}^{3} N_{i} .
$$

Equations [6] and [7] can be combined to express $\tau$ as a function of particle size,

$$
\begin{aligned}
\tau & =\frac{\phi K_{0} \pi}{(4 / 3) \pi}(\bar{a} / \lambda)^{m} \frac{\sum_{i} a_{i}^{2} N_{i}}{\sum_{i} a_{i}^{3} N_{i}} \\
& =\frac{3}{4} \phi K_{0} \frac{(\bar{a} / \lambda)^{m}}{a_{3 / 2}},
\end{aligned}
$$

where $a_{3 / 2}$ is the volume surface average particle radius.
Solving the equation for the ratio of initial turbidity, $\tau_{0}$, to the turbidity at any time, $\tau$, assuming no creaming (i.e. $\phi=\phi_{0}$ )

$$
\frac{\tau_{0}}{\tau}=\frac{\left(\bar{a}_{0} / \lambda\right)^{m_{0}}}{(\bar{a} / \lambda)^{m}} \frac{\left(a_{3 / 2}\right)}{\left(a_{3 / 2}\right)_{0}} .
$$

Assuming $\bar{a} / \bar{a}_{0}=\left(a_{3 / 2}\right) /\left(\left(a_{3 / 2}\right)_{0}\right)$ and substituting into Eq. [9],

$$
\frac{\tau_{0}}{\tau}=\frac{\left(\bar{a}_{0}\right)^{m_{0}-1} \lambda^{m-m_{0}}}{(\bar{a})^{m-1}} .
$$

In emulsion stability studies, it is desirable to determine the ratio of initial particle concentration to the particle concentration at any time, $N_{0} / N$, as a function of time. In the absence of any creaming the ratio $N_{0} / N$ can be related to $\tau_{0} / \tau$ using the relationship

$$
\frac{N_{0}}{N}=\frac{\bar{a}^{3}}{\bar{a}_{0}^{3}}
$$

Solving Eq. [10] for $\bar{a}$ and substituting in Eq. [11],

$$
\frac{N_{0}}{N}=\frac{1}{\bar{a}_{0}^{3}}\left[\frac{\lambda^{m-m_{0}}\left(\bar{a}_{0}\right)^{m_{0}-1}}{\tau_{0} / \tau}\right]^{3 / m-1} .
$$

For a special case when the particles are very large, $m_{0}=m=0$, Eq. [12] reduces to

$$
\frac{N_{0}}{N}=\left(\frac{\tau_{0}}{\tau}\right)^{3} \text {. }
$$

However, Dunn and Vold (1) used the relationship

$$
\frac{N_{0}}{N}=\frac{\tau_{0}}{\tau}
$$

to evaluate the effect of the molecular weight of a nonionic stabilizer on the stability of graphon dispersions. Lawrence and Parfitt (2) used the same method for measuring the stability of nonaqueous dispersions. They used an equation of the type

$$
\frac{-d \tau}{d t}=k^{\prime} \tau^{2}
$$

for

$$
-d N / d t=k N^{2}
$$


for kinetics of particle flocculation. Equation [12] can be used to determine the stability of an emulsion by measuring the initial average particle size, $\vec{a}_{0}$, and turbidity as a function of time. The values of $m$ are determined by measuring $\tau$ of the same sample at different $\lambda$ and plotting $\ln \tau$ vs $\ln \lambda$.

From the kinetics of flocculation

$$
-d N / d t=k N^{2},
$$

where $k=4 k T / 3 \mu \mathrm{W}$.

Integrating and using the initial condition $N=N_{0}$ at $\mathrm{t}=\mathbf{0}$,

$$
N_{0} / N=1+N_{0} k t .
$$

Therefore, by plotting $N_{0} / N$ vs $t$, one can determine the stability factor. The initial particle concentration and particle size is always required; however, it can be measured or it can be predicted from the method of preparation of the suspension. For example, the particle size of acoustically prepared emulsions can be estimated from the theory of $\mathrm{Li}$ and Fogler $(8,9)$.

The stability of acoustically prepared paraffin oil-in-water emulsions was determined by measuring the turbidity and initial particle size. An emulsion of $0.05 \mathrm{ml}$ $\mathrm{C}_{36} \mathrm{H}_{74}$ in $60 \mathrm{ml}$ water containing 4.2 $\times 10^{-3} \mathrm{~g}$ mole $\mathrm{MgSO}_{4} /$ liter was prepared by acoustic irradiation for $5 \mathrm{~min}$ at $80^{\circ} \mathrm{C}$ and stored at the same temperature. The initial particle size was determined by withdrawing a sample, freezing the particles, and placing them under a scanning electron microscope. Emulsion samples were drawn

\section{TABLE I}

Turbidity Data for Fig. $1^{t}$

\begin{tabular}{rcccr}
\hline $\begin{array}{r}\text { Time } \\
\text { (min) }\end{array}$ & $400 \mathrm{~nm}$ & $430 \mathrm{~nm}$ & $480 \mathrm{~nm}$ & \multicolumn{1}{c}{ Value of m } \\
\hline 0 & 0.344 & 0.330 & 0.300 & 0.459 \\
10 & 0.345 & 0.339 & 0.320 & 0.131 \\
20 & 0.301 & 0.304 & 0.300 & -0.239 \\
30 & 0.266 & 0.275 & 0.278 & -0.603 \\
45 & 0.232 & 0.240 & 0.243 & -0.610 \\
\hline
\end{tabular}

${ }^{n} N_{0}=3.61 \times 10^{9}$ particles $/ \mathrm{cm}^{3}$.

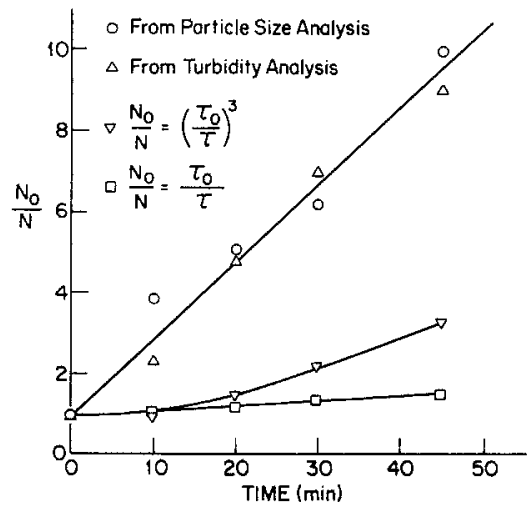

Fig. 1. Emulsion stability determined from the turbidity measurements.

at different times and diluted as required, and the turbidity was measured at three different wavelengths by using a Bausch and Lomb Spectronic 88 spectrophotometer.

The value of $m$ was obtained from the slope of the curve of a plot of $\ln \tau$ as a function of $\ln \lambda$. This curve was obtained from a polynomial fit of the values in Table I. The ratios $\left(N_{0} / N\right)$ at different times were calculated from Eq. [12] using a wavelength of $400 \mathrm{~nm}$ and the results are presented in Fig. 1.

For comparison, the ratio $N_{0} / N$ was also computed by measuring the particle size as a function of time as described below $(10,11)$. The total particle concentration, $N$, is related to the volumetric mean particle diameter, $D_{3 / 0}$. From a material balance on the oil,

$$
N=\frac{\phi}{(\pi / 6)\left(D_{3 / 0}\right)^{3}},
$$

where $\phi$ is volume fraction of oil, and $D_{3 / 0}$ is given by

$D_{3 / 0}=\left(f_{1} D_{i}^{3}+f_{2} D_{2}^{3}+f_{3} d_{3}^{3}+\cdots\right)^{1 / 3}$,

where $f_{1}$ is the fraction of particles in group $i$ with diameter $D_{i}$. Emulsion particles were divided into several groups with increment groupings of equal diameter. 


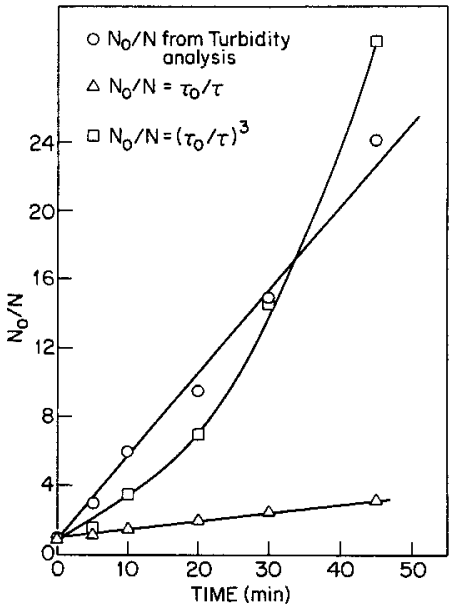

Frg. 2. Emulsion stability determined from different methods.

In the absence of creaming or sedimentation,

$$
\frac{N_{0}}{N}=\left[\frac{D_{3 / 0}}{\left(D_{3 / 0}\right)_{0}}\right]^{3}
$$

where subscript 0 indicates the initial value.

The values of $N_{0} / N$ from particle size measurements are also plotted in Fig. 1. The agreement between the two methods is excellent. Also plotted in Fig. 1 are $N_{0} / N$ calculated from Eqs. [13] and [14]. It is clear that one cannot approximate $N_{0} / N$ by $\tau_{0} / \tau$ or assume the particles are larger and $m=0$. Equation [12] should be used for

\section{TABLE II}

Turbidity Data for Fig. $2^{a}$

\begin{tabular}{rcccr}
\hline $\begin{array}{r}\text { Time } \\
\text { (min) }\end{array}$ & $400 \mathrm{~nm}$ & $450 \mathrm{~nm}$ & $500 \mathrm{~nm}$ & Values of $\mathrm{m}$ \\
\hline 0 & 0.574 & 0.552 & 0.524 & 0.255 \\
5 & 0.485 & 0.490 & 0.490 & -0.133 \\
10 & 0.380 & 0.395 & 0.403 & -0.402 \\
20 & 0.300 & 0.315 & 0.320 & -0.554 \\
30 & 0.235 & 0.250 & 0.260 & -0.606 \\
45 & 0.185 & 0.195 & 0.200 & -0.556 \\
\hline
\end{tabular}

${ }^{a} N_{0}=4.35 \times 10^{9}$ particles $/ \mathrm{cm}^{3}$. accurate determination of emulsion stability. Figure 2 is another case where the stability of an emulsion is determined by measuring the turbidity and the corresponding turbidity data are presented in Table II.

The turbidity method was used $(10,11)$ for investigating the stability and stability mechanism of acoustically formed emulsions. This simple and approximate method can be used for determining the stability of various other emulsions.

\section{SUMMARY}

The relationship between particle size and concentration and turbidity has been developed for a polydispersed system. The stability of acoustically prepared emulsions of $\mathrm{C}_{36} \mathrm{H}_{74}$ in water were determined from turbidimetry and found to be in agreement with the stability determined by the freezing method. The turbidimetry method can be used for determining the stability of various emulsions easily and inexpensively.

\section{REFERENCES}

1. Dunn, V., and Vold, R. D., J. Colloid Interface Sci. 54, 22 (1976).

2. Lawrence, S. G., and Parfitt, G. D., J. Colloid Interface Sci. 35, 675 (1971).

3. Bagchi, P., and Vold, R. D., J. Colloid Interface Sci. 33, 405 (1970).

4. Hiemenz, P. C., and Vold, R. D., J. Colloid Interface Sci. 21, 479 (1966).

5. Bagchi, P., and Vold, R. D., J. Colloid Interface Sci. 53, 194 (1975).

6. Weissberger, A. (Ed.), "Techniques of Organic Chemistry, Physical Methods of Organic Chemistry" Part III, pp. 2107-2145. Interscience, New York, 1960.

7. Lothian, G. F., and Chappel, F. P., J. Appl. Chem. 1, 475 (1951).

8. Li, M. K., and Fogler, H. S., J. Fluid Mech. 88, 499 (1978).

9. Li, M. K., and Fogler, H. S., J. Fluid Mech. 88, 513 (1978).

10. Reddy, S, R., "On the Stability of Emulsions." Ph.d. thesis, The University of Michigan, 1980.

11. Reddy, S. R. and Fogler, H. S., J. Phys. Chem. 84, 1570 (1980). 